\title{
Resonance-enhanced multiphoton ionization spectroscopy of laser-ablated copper atoms
}

\author{
School of Chemistry, University of Nottingham, University Park,
}

Nottingham NG7 2RD, UK

Anna Andrejeva, Joe P. Harris and Timothy G. Wright ${ }^{a}$

${ }^{\text {a }}$ Corresponding author. Email: Tim.Wright@nottingham.ac.uk

\begin{abstract}
Resonance-enhanced multiphoton ionization (REMPI) spectra of laser-ablated copper atoms entrained in a supersonic free jet expansion are reported. Depending on the ionization scheme employed, and the conditions under which the copper atoms are produced, very different spectra are produced, and these are discussed. In some circumstances, high proportions of metastable atoms survive the ablation and expansion process and are clearly seen in the spectra. The spectroscopic transitions for the observed lines are identified, and it is noted that some caution is merited in the assumption that only ground state copper atoms are present following laser ablation.
\end{abstract}




\section{Introduction}

The spectroscopy of copper has been studied extensively previously, with notable early studies by Shenstone ${ }^{1}$ (in emission) and Tondello ${ }^{2}$ (in absorption). The energy levels of neutral copper atoms have been discussed in detail by Martin and $\operatorname{Sugar}^{3}$ and subsequently by Connerade et. al. ${ }^{4}$ Additionally, Longmire et al. ${ }^{5}$ have looked closely at energy levels of neutral copper near the $3 \mathrm{~d}^{10}{ }^{1} \mathrm{~S}_{0}$ cationic ground state, which yielded a precise value of the ionization energy (IE) of $62317.44 \mathrm{~cm}^{-1}$. Baig et al. ${ }^{6}$ have reported comprehensive highresolution photoabsorption spectra of copper corresponding to transitions from the $3 \mathrm{~d}$ shell of the form $3 \mathrm{~d}^{9} 4 \mathrm{~s} n \mathrm{p}, n \mathrm{f}(n \geq 4) \leftarrow 3 \mathrm{~d}^{10} 4 \mathrm{~s}\left({ }^{2} S_{1 / 2}\right)$. In 1999, the Simard ${ }^{7}$ group reassessed the coinage metal first ionization energies and quantum defects using two-colour resonant excitation scheme, followed by forced ionization. More recently, the Bellert ${ }^{8}$ group revisited gaseous atomic copper recording $3 \mathrm{~d}^{10} n \mathrm{~d}\left({ }^{2} D_{J}\right) \leftarrow 3 \mathrm{~d}^{10} 4 \mathrm{~s}\left({ }^{2} S_{1 / 2}\right)(n=9-21)$ Rydberg transitions as well as those corresponding to $3 \mathrm{~d}^{10} 4 \mathrm{p}\left({ }^{2} P_{3 / 2,1 / 2}\right) \leftarrow 3 \mathrm{~d}^{10} 4 \mathrm{~s}\left({ }^{2} S_{1 / 2}\right)$. The abovementioned studies have been performed via excitation of copper from its ground electronic state, while very little work is available on the metastable state transitions. The latter data would be of great benefit, for example, in plasma science and also for the interaction of electrons leading to excited atoms. Diffusion coefficients for ground state, $3 \mathrm{~d}^{10} 4 \mathrm{~s}\left({ }^{2} S_{1 / 2}\right)$, as well as for metastable state, $3 \mathrm{~d}^{9} 4 \mathrm{~s}^{2}\left({ }^{2} D_{3 / 2}\right)$, of copper in both $\mathrm{Ne}$ and Ar have been measured by Sekido et al., ${ }^{9}$ who concluded that the diffusion coefficients were found to be nearly equal for these states at $300 \mathrm{~K}$. The only other study that we have found on metastable states of copper is by Shafran'osh and Snegurskaya who produced metastables by crossing a neutral $\mathrm{Cu}$ beam with an electron beam. ${ }^{10}$

In the present work, we examine observed REMPI transitions using UV radiation in the range $30,000-31,250 \mathrm{~cm}^{-1}$, noting that potentially more than one photon may be absorbed in reaching a resonant state. The copper atoms are produced by laser ablation, and are entrained in a pulse of rare gas subsequent to supersonic expansion into a vacuum chamber, prior to interaction with the excitation (and ionization) laser(s).

\section{Experimental}

The second harmonic (532nm, 5-6 mJ per pulse) of a neodymium-doped yttrium aluminium garnet (Nd:YAG) laser (Continuum Minilite II) was used to ablate metal atoms from the 
surface of a solid $\mathrm{Cu}$ rod (Goodfellow, 99.99\%), placed within a laser vaporization (LaVa) source made from polyether etherketone (PEEK), mounted on the high vacuum side of a pulsed nozzle. The initial design involved the $\mathrm{Cu}$ rod rotating and translating slowly, driven by a small four-phase stepper motor to ensure that a fresh surface of the rod is continually ablated, as this has worked well in previous work, including the production of Au-RG complexes $\left(\mathrm{RG}=\right.$ rare gas) from laser-ablated gold. ${ }^{11,12,13,14}$ However, in the present work, for copper this was found to give an unstable signal and after some investigation a stationary target was found to give a dramatic increase in signal stability, and so was used for the experiments reported here. Argon (BOC; 99.9+\%), held at pressure of 9-11 bar (a high backing pressure was employed as complexation experiments were being attempted, lower pressures were also employed, see later), was passed through a pulsed nozzle (Series 9, General Valve, $750 \mu \mathrm{m}$ orifice, $10 \mathrm{~Hz}$, opening time $\sim 270 \mu \mathrm{s}$ ) into a $1 \mathrm{~mm}$ channel within the LaVa source, where it flowed over the metal rod. The resulting hot, metal-containing plasma is collisionally cooled by the argon as it passes along a $1.5 \mathrm{~mm}$ diameter exit channel (length $20 \mathrm{~mm}$ ) before expansion into the nozzle chamber. The resulting supersonic jet expansion proceeds, unskimmed, into the extraction region of a time-of-flight mass spectrometer (located 30-40 cm downstream) located within the ionization chamber. In this chamber, the supersonic jet expansion is intercepted by the output of a tuneable dye laser (Sirah Cobra Stretch). The dye laser, operating on DCM dye in DMSO solvent, is pumped by the second harmonic (532 nm; 230mJ per pulse) of Surelite III Nd:YAG laser; the visible output was frequency doubled into the UV by a potassium dihydrogen phosphate (KDP) crystal, and the resulting UV radiation ( $3-5 \mathrm{~mJ}$ per pulse) is focused into the ionization chamber with a 30 $\mathrm{mm}$ focusing lens. For the two-colour experiments, ionization is achieved by overlapping, both spatially and temporally, the fourth harmonic ( $266 \mathrm{~nm}, \sim 4 \mathrm{~mJ}$ per pulse) of a Surelite I Nd:YAG laser with the frequency-doubled excitation laser output inside the ionization chamber. For both one- and two-colour experiments, the resulting ions are extracted into the time-of-flight mass spectrometer and detected by a dual microchannel plate (chevron) detector. Its signal is passed into an oscilloscope (LeCroy LT342 Waverunner) for monitoring, and additionally to a boxcar (SRS SR250) for integration and averaging; this signal is then relayed to a computer for storage and analysis.

The jet expansion, direction of propagation of the dye laser and axis of the time-of-flight mass spectrometer are arranged so as to be mutually perpendicular; when used, the $266 \mathrm{~nm}$ output was aligned counterpropagating to the frequency-doubled dye laser output. The timing 
of the three laser pulses relative to the opening time of the pulsed nozzle is achieved using digital delay generators (SRS DG535).

Calibration is achieved by employing a number of well-known $\mathrm{Cu}$ resonances. ${ }^{3}$ Relative wavenumber precision is estimated to be $\sim 0.4 \mathrm{~cm}^{-1}$; however, the breadth and asymmetry of the spectral features means that the band positions are less precisely determined.

\section{Results and Discussion}

a. $n$ d series.

Figure 1 shows spectra of copper atoms recorded under different REMPI regimes. In trace a, only the frequency-doubled output from the dye laser is present, and so this is a one-colour spectrum. It is relatively simple to assign the observed intense spectral lines to a $3 \mathrm{~d}^{10} n \mathrm{~d}$ Rydberg series, arising from a (2+1) REMPI process as indicated, which terminate at the first ionization energy, corresponding to the sharp step in signal seen to high wavenumber. We consistently saw lines corresponding to $n=8-16$, with a weak peak sometimes appearing in the correct position for $n=17$. Although generally the observed intensity distribution under these conditions is as shown, these were subject to the vagaries of the ablation process. The observed $3 \mathrm{~d}^{10} n \mathrm{~d}$ Rydberg line positions, $E_{\mathrm{n}}$, presented in Table 1 and compared to previously-reported values. Our values were fitted to the Rydberg equation (Eq. 1):

$$
E_{n}=I-\frac{R}{(n-\delta)^{2}} \quad \text { Eq. } 1
$$

where: $E_{n}$ is the energy of the Rydberg level with principal quantum number, $n$; $I$ is the ionization energy; $\delta$ is the quantum defect for the series; and $R$ is the Rydberg constant. We included the line positions for $n=8-16$ in the fit, but omitted $n=13$ as the position of its peak varied, caused by overlap with at least one other feature. In the nonlinear least squares fit, presented in Figure 2, $I$ and $\delta$ were obtained simultaneously, yielding a value of $62317(6)$ $\mathrm{cm}^{-1}$ for $I$ and 1.02(9) for $\delta$. Both of these $I$ and $\delta$ values are in very good agreement with the more-precise values of 62317.3(2) and 1.018(2) from Loock et al., ${ }^{7}$ and the even more precise values of $I$ available, $62317.44(10)^{3}$ and $62317.46(3) .{ }^{15}$ We note that a similar analysis by Castleberry et al. ${ }^{8}$ did not fit both $I$ and $\delta$ simultaneously, but fixed $I$ to the experimental value from ref. 3; this yielded a value of $\delta$ of $0.92(3)$, which is outside of the range from Loock et al. ${ }^{7}$ 
It is interesting to note the different ranges of the $n \mathrm{~d}$ series observed. In the present work, we saw up to $n=16$ clearly, with a weak feature for $n=17$ sometimes visible, while Castleberry et al. ${ }^{8}$ saw from $n=9$ up to $n=21$, with an intensity distribution similar to that in Fig. 1(a). Loock et al. ${ }^{7}$ observed the $n$ d series for $n=19-46$, with an intensity distribution that was markedly different to the present work or that of ref. 8; this difference lies in the detection method. In ref. 7, a two-colour, optical-optical double resonance, excitation scheme was used to access the $3 \mathrm{~d}^{10} n \mathrm{~d}$ (and $3 \mathrm{~d}^{10} n \mathrm{~s}$ ) Rydberg states, with the $3 \mathrm{~d}^{10} 4 \mathrm{p}^{1}\left({ }^{2} P_{1 / 2}\right)$ state being used as an intermediate level. The Rydberg states were then detected by forced ionization by application of a $500 \mathrm{~V}$ electric field; thus, a completely different detection method is employed in ref. 7 compared to that in the present work, or ref. 8 , and explains the very different intensities observed.

In principle, both spin-orbit components of the ${ }^{2} D_{5 / 2,3 / 2}$ Rydberg lines are accessible from the ground $3 \mathrm{~d}^{10} 4 \mathrm{~s}^{1}\left({ }^{2} S_{1 / 2}\right)$ state in a two-photon transition. ${ }^{16}$ The important selection rules for the two-photon processes include orbital angular momentum change $\Delta l=0, \pm 2$, where $l$ is the orbital angular momentum quantum number of the electron being excited ( $\mathrm{s} \leftarrow \mathrm{s}$ is also explicitly forbidden); whereas the total angular momentum change must be $\Delta J=0, \pm 1, \pm 2$, with the total electronic spin remaining unchanged if $L S$-coupling prevails. Thus, both ${ }^{2} D_{5 / 2}$ and ${ }^{2} D_{3 / 2}$ states should be accessible. However, the spin-orbit splitting at high $n$ number gets smaller $^{7,15}$ and is of the order of our laser linewidth or less, and so we are unable to resolve the two components in the present work.

\section{b. ${ }^{2} P_{\mathrm{J}} \leftarrow{ }^{2} S_{1 / 2}$ transition}

When $266 \mathrm{~nm}$ radiation is also present, then we find that two extra lines can be seen, which have a significant intensity (Fig. 1b). These can be identified as arising from the $3 \mathrm{~d}^{10} 4 \mathrm{p}^{1}$ $\left({ }^{2} P_{3 / 2,1 / 2}\right) \leftarrow 3 \mathrm{~d}^{10} 4 \mathrm{~s}^{1}\left({ }^{2} S_{1 / 2}\right)$ transition. By reducing the intensity of the tuneable UV radiation, it was possible to obtain a spectrum that is essentially wholly due to the ${ }^{2} P_{J} \leftarrow{ }^{2} S_{1 / 2}$ transitions observed via a $\left(1+1^{\prime}\right)$ ionization scheme - see Fig. 1c. This behaviour is observed since the $n \mathrm{~d} \leftarrow{ }^{2} S_{1 / 2}$ transitions occur at the two-photon level, but the $3 \mathrm{~d}^{10} 4 \mathrm{p}^{1}\left({ }^{2} P_{3 / 2,1 / 2}\right)$ levels lie less than half way to the first $\left(3 \mathrm{~d}^{10}\right)$ ionization limit, and as a consequence would require a $(1+2)$ REMPI process to yield ions, when only the frequency-doubled dye laser output is present; this is unfavourable compared to a $(2+1)$ process and very few of these ions are 
seen in a one-colour experiment. One the other hand, the $266 \mathrm{~nm}$ photon is able to ionize the ${ }^{2} P_{J}$ states directly.

It is interesting to note that over this wavenumber range, at the two-photon level, there are various $3 \mathrm{~d}^{10} n \mathrm{~s}$ and $3 \mathrm{~d}^{10} n \mathrm{p}$ Rydberg states. However, transitions to the $n$ s states are electric dipole forbidden at both the one- and two-photon level from the ground $3 \mathrm{~d}^{10} 4 \mathrm{~s}$ level, and transitions to the $3 \mathrm{~d}^{10} n \mathrm{p}$ states are electric dipole forbidden at the two-photon level. We see no firm evidence for these states, although we note that they would be expected to be weak (but see comment below), and that the $3 \mathrm{~d}^{10} n \mathrm{p}$ transitions become increasingly close in wavenumber to the $3 \mathrm{~d}^{10}(n-1) \mathrm{d}$ ones. ${ }^{3}$ We note that weak forbidden series have been seen by Longmire et al., ${ }^{5}$ using a large spectrograph.

\section{c. Metastables}

Figure $3 \mathrm{a}$ shows the same spectral region as in Figure 1, with the lines assigned to the $3 \mathrm{~d}^{10} n \mathrm{~d}$ $\left({ }^{2} D_{J}\right) \leftarrow 3 \mathrm{~d}^{10} 4 \mathrm{~s}\left({ }^{2} S_{1 / 2}\right)$ Rydberg transitions shown. In Figure 1a, it can be seen that there are a number of small lines in between the main Rydberg transitions; Figure 3a shows that these lines are much more prominent, with some lines reaching up to half the intensity of the ${ }^{2} D_{J}$ $3 \mathrm{~d}^{10} \mathrm{n} d$ Rydberg series lines. Figure $3 \mathrm{~b}$ shows a further increase in the intensities of these lines, where now some features are more intense than the $n$ d Rydberg series lines, particularly those between the $3 \mathrm{~d}^{10} 9 d$ and $3 \mathrm{~d}^{10} 10 d$ lines. Given the lack of other allowed transitions from the ground $3 \mathrm{~d}^{10} 4 \mathrm{~s}^{1}\left({ }^{2} S_{1 / 2}\right)$ state, we looked to metastable states as the initial level for these transitions.

In order for metastable states to arrive at the ionization region, they must be: produced during the ablation process; survive (or be produced in) the ablation plasma; survive (or be populated during) the passage down the channel from the ablation point; survive (or be populated during) the supersonic jet expansion; and also survive the journey to the ionization point. We estimate these metastables need to survive for at least about $600 \mu$ s to be observed at the front of the gas pulse, although these were observed at times suggesting lifetimes of $800 \mu$ s or longer. As such, they cannot be connected to a lower level by an allowed electronic transition, otherwise they would decay to it via fluorescence, and even states connected to lower states by spin-forbidden transitions are unlikely to survive this length of time. 
To assign the metastable transitions, we considered the lowest electronic states which would be expected to be metastable. Transitions to the ground state from the ${ }^{2} D_{J}$ levels, arising from the $3 \mathrm{~d}^{9} 4 \mathrm{~s}^{2}$ configuration, are not electric dipole allowed, since they arise from a $\mathrm{s} \leftarrow \mathrm{d}$ transition. These are thus expected to be reasonably long lived; the ${ }^{2} D_{5 / 2}$ level is expected to be the longer lived, since transitions to the ground state are also forbidden even if $J j$ coupling is considered, while the ${ }^{2} D_{3 / 2}$ level could potentially relax to the ${ }^{2} S_{1 / 2}$ ground state, in a $\Delta J=1$ transition. The next set of states are the ${ }^{4} P_{\mathrm{J}}$ and ${ }^{4} F_{\mathrm{J}}$ states arising from a $3 \mathrm{~d}^{9} 4 \mathrm{~s} 4 \mathrm{p}$ configuration. Transitions from these states to the ground state are spin-forbidden. The ${ }^{4} P_{J}$ states are lower in energy, and have $J=1 / 2-5 / 2$; as such, these could decay to the lower ${ }^{2} D_{J}$ levels via $\Delta J=0,1$ processes. The ${ }^{4} F_{J}$ states overlap the ${ }^{4} P_{J}$ ones and have $J=3 / 2-9 / 2$. Again, $\Delta J=0,1$ transitions to lower states could occur for the ${ }^{4} F_{3 / 2,5 / 2,7 / 2}$ levels, with the ${ }^{4} F_{9 / 2}$ level expected to be the longest lived, since it requires $\Delta J=2$ to attain the ground state. In Figure 3, we have labelled the most prominent transitions A-F; however, we note that there are other weak transitions in the spectra, and indeed in various other spectra we have recorded, other weak features can be seen - we shall comment on these briefly below.

In Table 2, we give the assignments for these features. As may be seen, these are dominated by transitions from the $3 \mathrm{~d}^{9} 4 \mathrm{~s}^{2}\left({ }^{2} D_{5 / 2}\right)$ state, with both one- and two-photon transitions observed. We note that we did attempt power-dependence studies, but these proved to be inconclusive, owing to variations in the ablated copper signal. Transitions from the ${ }^{4} F_{9 / 2}$ level are also observed, and these can only be one-photon in nature for this wavenumber range. Of note is that features $\mathrm{A}$ and $\mathrm{C}$ both are assigned as having more than one contribution, as these are close enough in wavenumber not to be resolved in the present work. We note that the observation of the ${ }^{2} D_{5 / 2}$ and ${ }^{4} F_{9 / 2}$ states by REMPI requires ionization to the second ionization limit, which lies just under $22,000 \mathrm{~cm}^{-1}$ above the first ionization limit, ${ }^{3}$ and so is indeed accessible using an additional photon in each case.

Although we are cautious regarding relative intensity changes, we note that bands $\mathrm{A}, \mathrm{C}$ and $\mathrm{F}$ appear to be more intense in Figure $3 \mathrm{~b}$ than in Figure $3 \mathrm{a}$, relative to $n$ d lines. Since feature $\mathrm{F}$ only arises from a single transition from the $3 \mathrm{~d}^{9} 4 \mathrm{~s}^{2}\left({ }^{2} D_{5 / 2}\right)$ state, the implication is that there is a higher population of this level under the conditions in which Figure $3 \mathrm{~b}$ was recorded; this would tie in with both bands $\mathrm{A}$ and $\mathrm{C}$ also increasing in intensity. Feature $\mathrm{B}$ also arises from this level, and also increases. We note that band $\mathrm{E}$ only arises from the ${ }^{4} \mathrm{~F}_{9 / 2}$ state, and its intensity is surpassed by both $\mathrm{C}$ and $\mathrm{F}$ in Figure $3 \mathrm{~b}$, while it was more intense in Figure $3 \mathrm{a}$. 
Thus, unsurprisingly, the relative populations of the ${ }^{2} D_{5 / 2}$ and ${ }^{4} F_{9 / 2}$ levels change, depending on the ablation conditions.

It is noteworthy that one of the assigned transitions from the ${ }^{2} D_{5 / 2}$ level for each of the lines $\mathrm{C}$ and $\mathrm{F}$ are two-photon and involve $\mathrm{p} \leftarrow \mathrm{s}$ excitations, which are forbidden by the two-photon $\Delta l=0, \pm 2$ selection rule. Although band $\mathrm{C}$ also has a fully-allowed one-photon contribution, which is expected to dominate the observed feature, band F only has the two-photon contribution. Such $\mathrm{p} \leftarrow \mathrm{s}$ transitions could be allowed by a breakdown of the $\Delta l$ rule, for example via magnetic quadrupole or electric octapole effects, but it is perhaps surprising that these are observed so strongly. It is interesting to note that the one-photon forbidden $7 \mathrm{~s} \leftarrow 4 \mathrm{~s}$ and $8 \mathrm{~s} \leftarrow 4$ s transitions were observed to be relatively intense by Shenstone, ${ }^{1}$ although other such $n$ s $\leftarrow 4$ s transitions were not seen (higher- $n$ transitions were only seen with a sensitive instrument ${ }^{5}$ ). It seems clear that there are interesting interstate couplings in copper, localized in narrow wavenumber regions, which are not yet fully understood, leading to $J$ states with mixed character; mixing of states involved in two-photon-allowed transitions would provide routes for intensity borrowing.

As mentioned above, numerous other weak/overlapping transitions were observed in the present work, and many of these could be assigned to various transitions from the ${ }^{2} D_{5 / 2}$ state. Some, however, were assignable as arising from the ${ }^{2} D_{3 / 2}$ state, with some close to and past the first ionization step being quite intense. Similarly, a few seemed to arise from the lowest ${ }^{4} P_{J}$ states and others of the lowest ${ }^{4} F_{J}$ states. Since these states are metastable but expected not to be long-lived (as there are allowed $\Delta J$ transitions to the ground state), a possible explanation is that their observation arise from a cascade of optical decays that result in their population, with the extent observed dependent on the nascent populations and other processes occurring in the ablation source. In a similar way, the population of the lowest ${ }^{2} D_{5 / 2}$ and ${ }^{4} F_{9 / 2}$ metastable states can also be produced in this way, but these states are long-lived enough to have a sizeable population under a wider range of conditions.

One feature requires further comment, which has been marked with an asterisk in Figure 3 and is at $30159.6 \mathrm{~cm}^{-1}$. This line can be seen to be more intense in Figure 1a, between the $8 \mathrm{~d}$ and $9 \mathrm{~d}$ lines, where it is also marked with an asterisk. We were unable to find a suitable assignment for this line, but note that it was seen very weakly by Shenstone, who attributed it to the following transition: $3 \mathrm{~d}^{9} 4 \mathrm{~s} 6 \mathrm{~s}\left({ }^{2} D_{3 / 2}\right) \leftarrow 3 \mathrm{~d}^{9} 4 \mathrm{~s} 4 \mathrm{p}\left({ }^{2} D_{3 / 2}\right)$, although we note that the lower level at $46172.84 \mathrm{~cm}^{-1}$ is noted as having mixed character in Sugar and Musgrove. ${ }^{3}$ We note 
that, although we recorded many spectra like Figure 1a where this line was clearly visible, numerous others were like those in Figure 3, where this feature was very weak. We were able to identify several other weak features which could originate from the same lower level, but we note that this state is not expected to be particularly long-lived and so its appearance in our spectra is somewhat surprising. Again, it could be populated via a cascade of optical transitions, and indeed we see a few weak features that could be assignable as originating in the two lowest ${ }^{2} P_{J}$ states; we note that the $n$ d Rydberg states could fluoresce to this level, for example, and this could populate lower levels within the laser interaction region, which are then then excited and ionized.

\section{Further Comments and Conclusions}

The presence of such intense lines from metastable states in this spectral region is surprising, since this does not appear to have been commented on previously. Additionally, in our earlier work on gold complexes, ${ }^{11,12,13,14}$ we did not experience any such problems, which could be due to a combination of such transitions for gold being in a different spectral region from the strong ${ }^{2} P_{J} \leftarrow{ }^{2} S_{1 / 2}$ transitions, and the latter being observable by one-colour $(1+1)$ REMPI, rather than necessitating either $(1+2)$ one-colour, or $\left(1+1^{\prime}\right)$ two-colour REMPI as in the case of copper. We also think that there are other factors, arising from the greater difficulty of ablating copper; gold is, of course, softer than copper, and so is expected to ablate more easily. Other workers have reported difficulties in ablating copper, ${ }^{17}$ and so have employed a different production method: dc discharge. ${ }^{17,18}$ In the present work, we have obtained stable copper signals by ablation for many hours, but we have found that the conditions are very sensitive, and a small change in conditions can suddenly lead to an erratic signal, or even a loss of signal. Part of this may be due to the monitoring, as stability is usually judged by sitting on a particular transition; as we have shown in this work, changing the ablation conditions can lead to a change in the observed state distribution, and so a change in a particular state's population. We did attempt a series of systematic studies to try and understand the metastable production in more detail; however, it proved difficult to obtain conclusive results. Based on the observations and a consideration of the experimental conditions, we hypothesise that significant metastable production is reliant on the balance between: (i) producing enough $\mathrm{Cu}$ atoms overall (requiring high ablation laser power densities, and so potentially more metastables in a hotter plasma); (ii) the pressure of the 
backing gas, which will increase the number of collisions of Ar with $\mathrm{Cu}^{*}$; and (iii) the length of the post-ablation channel (which will allow more deactivating collisions). All of these conditions were varied as well as the point at which the ablation plasma entered the Ar gas pulse, and also attempts at free ablation into the jet expansion (i.e. without a post-ablation channel). In future experiments, we will investigate the effect of varying the wavelength of the ablation laser.

It is also interesting to note that the spectrum of the $\mathrm{Cu}-\mathrm{Kr}$ complex, reported as arising via the ${ }^{2} P_{J}$ states, seems to have been recorded via a one-colour REMPI process. ${ }^{19}$ In the present work, we have shown that it is difficult to get strong atomic transitions from the ground state using a one-colour process in this wavenumber region, although one-photon, one-colour metastable signals can be observed, as can two-photon, one-colour $n$ d Rydberg transitions, under various conditions. We have noted previously ${ }^{20}$ that the unexpectedly high reported binding energy of the ground state $\mathrm{Cu}-\mathrm{Kr}$ complex, derived from the excited state values, could be due to metastable states of $\mathrm{Cu}$ being present. The current results suggest this hypothesis should be investigated further. Fewer problems from metastables may be experienced when the resultant copper atoms are reacted to form strongly-bound molecules, since here various routes to forming ground state species are likely to exist, particularly if spin-orbit coupling is significant. For example, we found it facile to replicate a number of transitions arising from $\mathrm{CuO}$ as recently reported by Mackenzie and coworkers, who also used laser ablation to produce the copper atoms. ${ }^{21}$

In summary, the ablation of copper can lead to the production of significant quantities of metastable atoms, as well as of the ground state. Caution should therefore be exercised in the assumption that only ground state atoms are present. 


\section{Acknowledgements}

The EPSRC are thanked for funding (Grant EP/I012303/1), and the provision of a studentship to J. P. H.; A.A. is grateful to the University of Nottingham and the EPSRC for the provision of a DTA studentship. 


\section{Figure Captions:}

Figure 1. REMPI spectra of copper atoms recorded under different ionization conditions. (a) One-colour $(2+1)$ spectrum showing the strong $3 \mathrm{~d}^{10} n \mathrm{~d}$ Rydberg series. The step to high wavenumber corresponds to the first ionization energy. The weak features in between the Rydberg series lines are assigned to transitions from metastable atoms - see text. (b) Conditions where the excitation laser is still relatively intense, but now $266 \mathrm{~nm}$ radiation is also present, allowing two-colour, $\left(1+1^{\prime}\right)$, REMPI to occur. (c) The excitation laser power has now been turned down so that very little one-colour signal is seen, and so ionization occurs mostly from the $266 \mathrm{~nm}$ radiation.

Figure 2. Graph of the experimental band positions of the ${ }^{2} D_{5 / 2} 3 \mathrm{~d}^{10} n \mathrm{~d}$ Rydberg series plotted against $n$. The line is the non-linear least squares fit, with the points being the experimental line positions. Note that $n=13$ was not included in the fit as its spectral line is overlapped.

Figure 3. REMPI spectra showing strong metastable lines. In trace $b$, the transitions involving metastables are more intense than those in trace a, and indeed they are more intense than the $n$ d Rydberg series. (Compare these spectra with those in Figure 1a.) See discussion in Section 4 regarding conditions. 
Figure 1.

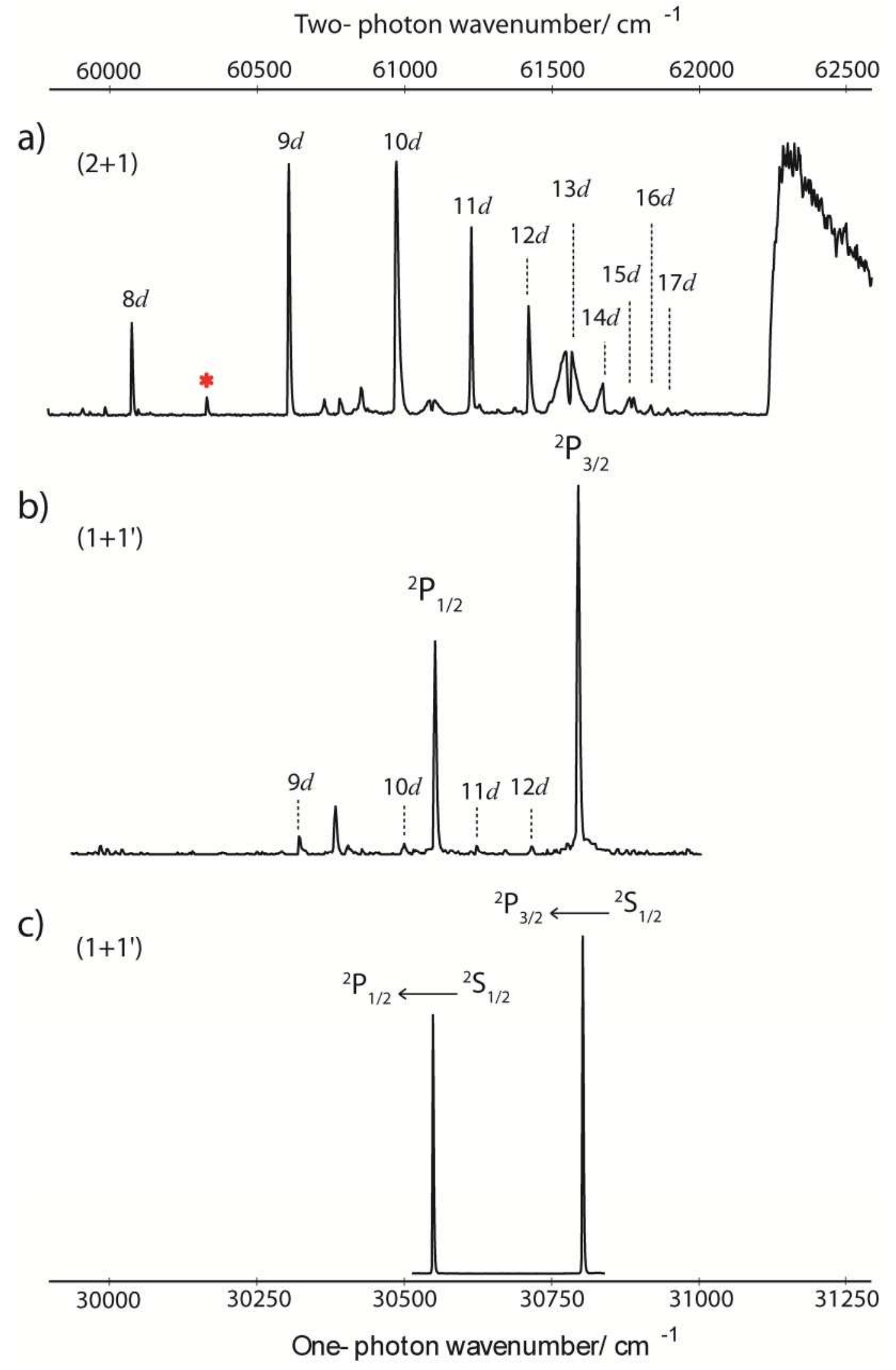


Figure 2.




Figure 3.

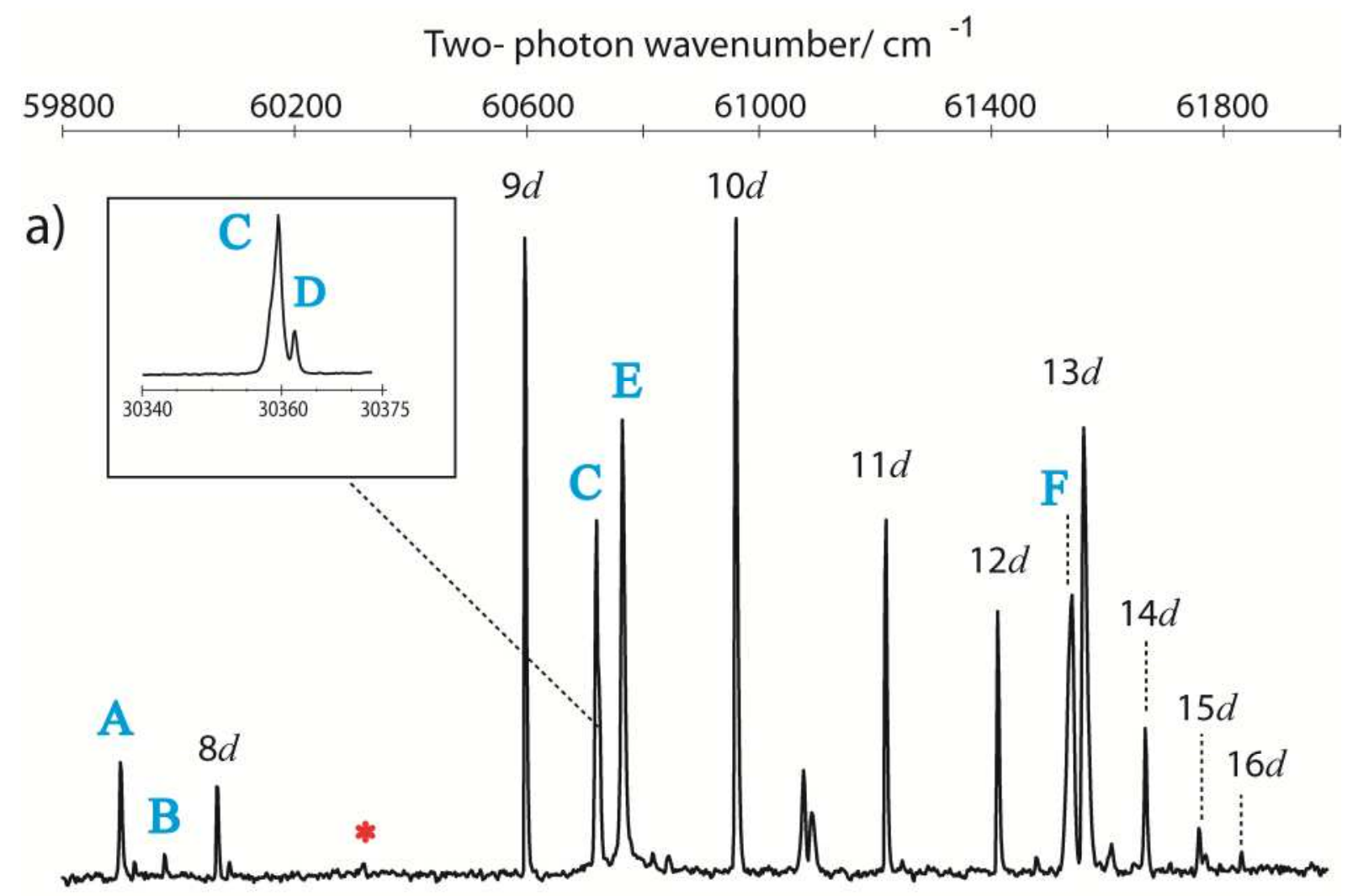

b)

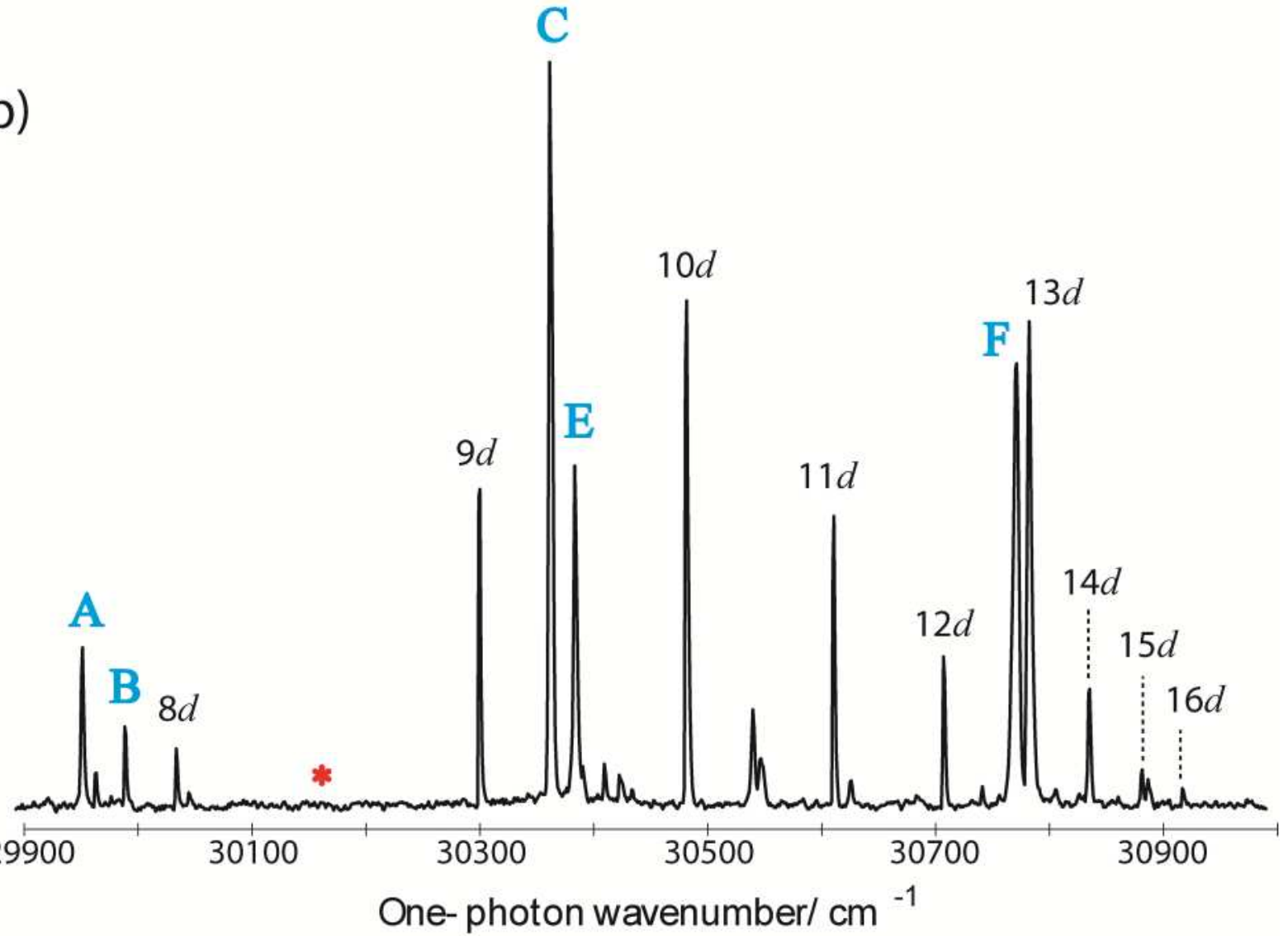


Table 1: Measured line positions of the $n$ d Rydberg series $(n=8-17)$ from the present study and those from previous work.

\begin{tabular}{|c|c|c|c|c|}
\hline Band Assignment & Line position $\left(\mathrm{cm}^{-1}\right)^{\mathrm{a}}$ & Ref. 3 & Ref. 8 & Ref. $7^{\mathrm{b}}$ \\
\hline $3 \mathrm{~d}^{10} 8 \mathrm{~d}$ & 60065.6 & 60066.33 & & 60066.19 \\
\hline $3 \mathrm{~d}^{10} 9 \mathrm{~d}$ & 60596.0 & 60595.05 & 60595.6 & 60594.90 \\
\hline $3 \mathrm{~d}^{10} 10 \mathrm{~d}$ & 60957.2 & 60957.35 & 60961.4 & 60957.07 \\
\hline $3 \mathrm{~d}^{10} 11 \mathrm{~d}$ & 61214.8 & 61215.59 & 61216.0 & 61215.96 \\
\hline $3 \mathrm{~d}^{10} 12 \mathrm{~d}$ & 61407.2 & & 61408.0 & 61407.39 \\
\hline $3 \mathrm{~d}^{10} 13 \mathrm{~d}^{\mathrm{c}}$ & 61554.0 & & 61557.4 & 61552.93 \\
\hline $3 \mathrm{~d}^{10} 14 \mathrm{~d}$ & 61661.6 & & 61662.8 & 61666.15 \\
\hline $3 \mathrm{~d}^{10} 15 \mathrm{~d}$ & 61754.4 & & 61754.6 & 61755.96 \\
\hline $3 \mathrm{~d}^{10} 16 \mathrm{~d}$ & 61825.2 & & 61826.6 & 61828.40 \\
\hline $3 \mathrm{~d}^{10} 17 \mathrm{~d}$ & 61883.2 & & 61885.4 & 61887.66 \\
\hline
\end{tabular}

${ }^{\mathrm{a}}$ This work

${ }^{\mathrm{b}}$ Line positions calculated using Equation 1 and the values of $I$ and $\delta$ given in ref. 7.

${ }^{\mathrm{c}}$ Line position affected by overlapping feature. 
Table 2: Copper atom metastable line positions and assignments. Initial and final state wavenumbers taken from ref. 3.

\begin{tabular}{|c|c|c|c|c|c|}
\hline $\begin{array}{l}\text { Spectral } \\
\text { Feature }\end{array}$ & $\begin{array}{l}\text { Position } \\
\text { (one- } \\
\text { photon) / } \\
\mathrm{cm}^{-1}\end{array}$ & $\begin{array}{l}\text { Photon } \\
\text { order }\end{array}$ & Assignment & $\begin{array}{c}\text { Final state } \\
\text { wavenumber } / \mathrm{cm}^{-1}\end{array}$ & $\begin{array}{c}\text { Initial state } \\
\text { wavenumber } / \mathrm{cm}^{-1}\end{array}$ \\
\hline $\mathrm{A}$ & 29950.0 & $\begin{array}{l}1 \\
1 \\
\end{array}$ & $\begin{array}{c}3 \mathrm{~d}^{9} 4 \mathrm{~s} 4 \mathrm{p}\left({ }^{4} F_{7 / 2}\right) \leftarrow 3 \mathrm{~d}^{9} 4 \mathrm{~s}^{2}\left({ }^{2} D_{5 / 2}\right) \\
3 \mathrm{~d}^{9} 4 \mathrm{~s} 4 \mathrm{~d}\left({ }^{2} G_{9 / 2}\right) \leftarrow 3 \mathrm{~d}^{9} 4 \mathrm{~s} 4 \mathrm{p}\left({ }^{4} F_{9 / 2}\right)\end{array}$ & $\begin{array}{c}41153.433 \\
70859.53 \\
\end{array}$ & $\begin{array}{c}11202.57 \\
40909.138 \\
\end{array}$ \\
\hline $\mathrm{B}$ & 29988.2 & 2 & $3 \mathrm{~d}^{9} 4 \mathrm{~s} 4 \mathrm{~d}\left({ }^{4} P_{5 / 2}\right) \leftarrow 3 \mathrm{~d}^{9} 4 \mathrm{~s}^{2}\left({ }^{2} D_{5 / 2}\right)$ & 71178.19 & 11202.57 \\
\hline $\mathrm{C}$ & 30359.7 & $\begin{array}{l}1 \\
2 \\
1\end{array}$ & $\begin{aligned} & 3 \mathrm{~d}^{9} 4 \mathrm{~s} 4 \mathrm{p}\left({ }^{4} F_{5 / 2}\right) \leftarrow 3 \mathrm{~d}^{9} 4 \mathrm{~s}^{2}\left({ }^{2} D_{5 / 2}\right) \\
& 3 \mathrm{~d}^{9} 4 \mathrm{~s} 4 \mathrm{p}\left({ }^{2} P_{3 / 2}\right) \leftarrow 3 \mathrm{~d}^{9} 4 \mathrm{~s}^{2}\left({ }^{2} D_{5 / 2}\right) \\
& 3 \mathrm{~d}^{9} 4 \mathrm{~s} 4 \mathrm{~d}\left({ }^{4} D_{7 / 2}\right) \leftarrow 3 \mathrm{~d}^{9} 4 \mathrm{~s} 4 \mathrm{p}\left({ }^{4} F_{9 / 2}\right)\end{aligned}$ & $\begin{array}{c}41562.895 \\
71917 \\
71268.21\end{array}$ & $\begin{array}{c}11202.57 \\
11202.57 \\
40909.138\end{array}$ \\
\hline $\mathrm{D}$ & 30362.1 & 2 & $3 \mathrm{~d}^{9} 4 \mathrm{~s} 4 \mathrm{~d}\left({ }^{4} P_{3 / 2}\right) \leftarrow 3 \mathrm{~d}^{9} 4 \mathrm{~s}^{2}\left({ }^{2} D_{5 / 2}\right)$ & 71927.22 & 11202.57 \\
\hline $\mathrm{E}$ & 30381.9 & 1 & $3 \mathrm{~d}^{9} 4 \mathrm{~s} 4 \mathrm{~d}\left({ }^{4} F_{9 / 2}\right) \leftarrow 3 \mathrm{~d}^{9} 4 \mathrm{~s} 4 \mathrm{p}\left({ }^{4} F_{9 / 2}\right)$ & 71290.54 & 40909.138 \\
\hline $\mathrm{F}$ & 30767.3 & 2 & $3 \mathrm{~d}^{9} 4 \mathrm{~s} 5 \mathrm{p}\left({ }^{2} P_{1 / 2}\right) \leftarrow 3 \mathrm{~d}^{9} 4 \mathrm{~s}^{2}\left({ }^{2} D_{5 / 2}\right)$ & 72737.8 & 11202.57 \\
\hline
\end{tabular}




\section{References}

${ }^{1}$ A. G. Shenstone, Phil Trans. R. Soc. Lond. A, 241, 297 (1948).

${ }^{2}$ G. Tondello, J.Opt. Soc. Am., 63, 346 (1973).

${ }^{3}$ J. Sugar and A. Musgrove, J. Phys. Chem. Ref. Data, 19, 529 (1990).

${ }^{4}$ J. P. Connerade, M. A. Baig, M. W. D. Mansfield and E. Redtke, Proc. R. Soc. A, 361, 379 (1978).

${ }^{5}$ M. S. Longmire, C. M. Brown and M. L. Ginter, J. Opt. Soc. Am., 70, 423 (1980).

${ }^{6}$ M. A. Baig, M. Hanif, S. A. Bhatti and J. Hormes, J. Phys. B: At. Mol. Opt. Phys., 30, 5381 (1997).

${ }^{7}$ H. Loock, L. M. Beaty and B. Simard, Phys. Rev. A, 59, 873 (1999).

${ }^{8}$ V. Castleberry, J. Dee, O. Villarroel, I. Laboren and D. Bellert, Phys. Lett A, 372, 4805 (2008).

${ }^{9}$ H. Sekido, T. Kondo, A. Kono and T. Goto, J. Phys. D: Appl. Phys., 26, 1414 (1993).

${ }^{10}$ I. I. Shafran'osh and T. A. Snegurskaya, Opt. and Spec., 86, 171 (1999).

${ }^{11}$ R. J.Plowright, V. L. Ayles, M. J.Watkins, A. M. Gardner, R. R. Wright, T. G. Wright and W. H. Breckenridge J. Chem. Phys. 12, 204308(2007).

${ }^{12}$ R. J. Plowright, M. J. Watkins, A. M. Gardner, T. G. Wright, W. H. Breckenridge, F. Wallimann and S. Leutwyler J. Chem. Phys. 129, 154315 (2008).

${ }^{13}$ R. J. Plowright, M. J. Watkins, A. M. Gardner, C. D. Withers, T. G. Wright and W. H. Breckenridge Phys. Chem. Chem. Phys. 11, 1539 (2009).

${ }^{14}$ R. J. Plowright, A. M. Gardner, C. D. Withers, T. G. Wright, M. D. Morse and W. H. Breckenridge J. Phys. Chem. A 114, 3103 (2010).

${ }^{15}$ K. B. MacAdam, S. F. Dyubko, V. A. Efremov, V. G. Gerasimov and A. S. Kutsenko, J. Phys. B. 42, 165009 (2009).

${ }^{16}$ See, for example, M. N. R. Ashfold, S. G. Clement, J. D> Howes and C. M. Western, J. Chem. Faraday Trans. 89, 1153 (1993).

${ }^{17}$ F. X. Sunahori, X. Zhang and D. J. Clouthier, J. Chem. Phys. 125, 084310 (2006).

${ }^{18}$ A. Janczyk, S. K. Walster, and L. M. Ziurys, Chem. Phys. Lett, 401, 211 (2005).

${ }^{19}$ L. R. Brock and M. A Duncan, Chem. Phys. Lett. 247, 18 (1995).

${ }^{20}$ A. M. Gardner, R. J. Plowright, M. J. Watkins, T. G. Wright and W. H. Breckenridge

J. Chem. Phys. 132, 184301 (2010).

${ }^{21}$ I. S. Parry, A. C. Hermes, A. Kartouzian and S. R. Mackenzie, Phys. Chem. Chem. Phys. 16, 458 (2014). 